\title{
Backup ventilation during neurally adjusted ventilatory assist in preterm infants
}

\author{
JUYOUNG LEE ${ }^{1}$, Vilhelmiina Parikka ${ }^{2}$, Liisa Lehtonen ${ }^{2}$, and Hanna Soukka² \\ ${ }^{1}$ Inha University College of Medicine \\ ${ }^{2}$ TYKS Turku University Hospital
}

May 18, 2021

\begin{abstract}
Objective: To analyze the proportion of backup ventilation during neurally adjusted ventilatory assist (NAVA) in preterm infants at different gestational ages and to analyze the trends in backup ventilation in relation to clinical deteriorations. Methods: A prospective observational study was conducted in 18 preterm infants born at a median (range) $27^{+4}\left(23^{+4}-34^{+4}\right.$ ) weeks of gestation with a median (range) birth weight of 1,100 (460-2,820) g, who received respiratory support with either invasive or noninvasive NAVA. Data on ventilator settings and respiratory variables were collected daily; the mean values of each 24-hour recording were computed for each respiratory variable. For clinical deterioration, ventilator data were reviewed at 6-hour intervals for 30 hours prior to the event. Results: A total of 354 ventilator days were included: 269 and 85 days during invasive and noninvasive NAVA, respectively. The time on backup ventilation $(\% / \mathrm{min})$ significantly decreased, and the neural respiratory rate increased with increasing postmenstrual age during both invasive and noninvasive NAVA. The median time on backup ventilation was less than $15 \% / \mathrm{min}$, and the median neural respiratory rate was more than 45 breaths/min for infants above $26^{+0}$ weeks of gestation during invasive NAVA. The relative backup ventilation significantly increased prior to the episode of clinical deterioration. Conclusion: The proportion of backup ventilation during NAVA showed how the control of breathing matured with increasing gestational age. Even the most immature infants triggered most of their breaths by their own respiratory effort. An acute increase in the proportion of backup ventilation anticipated clinical deterioration.
\end{abstract}

\section{Introduction}

Neurally adjusted ventilatory assist (NAVA) has been developed and evaluated in many studies, including studies of preterm infants, over the last decade. This technique uses the electrical activity of the diaphragm (Edi) to control respiratory support and the NAVA level as an amplification factor that converts the Edi signal into a proportional pressure. ${ }^{1}$ NAVA synchronizes mechanical breaths with the patient's neural respiratory drive and supports this drive proportionally to its effort. ${ }^{1-3}$ Several studies have demonstrated that the patient-ventilator interaction is improved and that delivered pressure is decreased with NAVA compared with other conventional modes in preterm infants. ${ }^{4-9}$

NAVA uses the patient's own respiratory effort as both a trigger and an assist control during respiratory support. ${ }^{1,10}$ Because of these characteristics, many clinicians are concerned if the weak respiratory effort of preterm infants would hinder NAVA working efficiently for them. ${ }^{11-14}$ This is one of the important obstacles causing hesitation regarding the use of NAVA in preterm infants. Additionally, there is a paucity of research on the use of NAVA in the most immature preterm infants.

We aimed to investigate the success of NAVA ventilation at different gestational ages in preterm infants by analyzing the proportion of backup ventilation during NAVA. In addition, the trends in backup ventilation were analyzed in relation to clinical deteriorations.

\section{Materials and Methods}


A prospective observational study took place from March 2020 to December 2020 in the level III neonatal intensive care unit of Turku University Hospital, Finland. We included preterm infants born before 36 weeks of gestation who received at least 24 hours of ventilatory support with invasive or noninvasive NAVA using Servo-i or Servo-n ventilators (Getinge, Solna, Sweden).

As NAVA was the primary ventilation mode in this unit, for intubated preterm infants, we began NAVA whenever the infants had spontaneous breathing. As soon as possible we extubated them and applied noninvasive NAVA. Before the start of NAVA mode, a standard feeding tube was replaced with a dedicated electrode-equipped catheter to detect Edi (Edi catheter, Getinge, Solna, Sweden). The trigger Edi level was set as $0.5 \mu \mathrm{V}$ above the minimum Edi. Attending clinicians decided and adjusted the NAVA level, positive end-expiratory pressure (PEEP), fraction of inspired oxygen $\left(\mathrm{FiO}_{2}\right)$ and backup ventilation (pressure control mode) settings according to the infant's condition. The apnea time was set as 2 to 10 seconds. After the set apnea time without the Edi signal, the backup ventilation started with a preset peak pressure and frequency until the Edi trigger resumed. Our usual backup rate was set as 20 to 40 breaths/min and the inspiratory time was set as 0.4 to 0.5 seconds. The safety limit for peak inspiratory pressure (PIP) was set as 25 to 35 $\mathrm{cmH}_{2} \mathrm{O}$. If the infant lost his/her cardiorespiratory stability during NAVA, the ventilator mode was changed, and this episode was defined as 'clinical deterioration.'

For the infant participating in the study, ventilator settings and respiratory variables were recorded every day and exported to a specific computer using Servo Record Viewer version 1.0 (Maquet Critical Care AB, Getinge, Solna, Sweden). The collected data provided the values for PIP, mean airway pressure, PEEP, expiratory tidal volume, peak Edi, minimum Edi, measured respiratory rate (RR), neural respiratory rate (nRR) and percentage time spent in backup ventilation for each minute. All the ventilator data were inspected and compared with the event logs recorded automatically from the ventilator, which included all alarm notifications, mode and setting changes, cable connections and disconnections. Data during disconnection of the Edi cable, malfunction or dislocation of the Edi catheter were excluded from the analysis. The mean values during each day were computed for each ventilatory variable. If there was a change in the ventilatory setting parameters, we chose the parameter that was applied for a longer duration in the 24-hour time period. For clinical deterioration, ventilator data 30 hours prior to the episode were reviewed at 6 -hour intervals. The zero time point was defined as the moment when the ventilator mode was switched from NAVA to others because of clinical deterioration.

The following data were collected from medical records: gestational age at birth, birth weight, sex, postmenstrual age (PMA) and body weight at the study date. For clinical deterioration, information about its cause and interventions were collected. The study was approved by the Ethics Committee of the Hospital District of Southwest Finland as well as by the scientific research committee of the Pediatric Department of Turku University Hospital, Finland. Written informed consent was obtained from both parents according to the guidelines of the Research and Ethics Board before patient enrollment in this study.

Correlations between respiratory parameters and PMA were analyzed using the Spearman test. Changes in the proportion of backup ventilation before clinical deterioration were analyzed using the Friedman test. Ap value $<0.05$ was considered to be statistically significant. Statistical analyses were performed using SPSS v27.0 (IBM, Armonk, NY, USA).

\section{Results}

Eighteen preterm infants born at a median (range) $27^{+4}\left(23^{+4}-34^{+4}\right)$ weeks of gestation with a median (range) birth weight of 1,100 (460-2,820) g were included in the study. A total of 354 patient days of ventilator data were collected: 269 patient days during invasive NAVA and 85 patient days during noninvasive NAVA. The percentage of excluded data was a median (IQR) of $0.8 \%(0.4-1.6 \%)$ per each patient day: $0.9 \%(0.4-$ $1.7 \%)$ during invasive NAVA and $0.7 \%(0.3-1.0 \%)$ during noninvasive NAVA. Baseline characteristics and ventilator settings at the time of data collection are described in Table 1. On the day of data collection, the infant's age in days, PMA and body weight ranged from 1 to 90 days, $24^{+2}$ to $36^{+6}$ weeks and 550 to 3,260 $\mathrm{g}$, respectively. Their median (range) NAVA level was $1.5(0-2.5) \mathrm{cmH}_{2} \mathrm{O} / \mu \mathrm{V}$. The preset median (range) 
apnea time and backup ventilation during apnea were $3(2-10)$ seconds and $30(16-40)$ breaths per minute, respectively (Table 1 ).

During both invasive and noninvasive NAVA, the time on backup ventilation $(\% / \mathrm{min})$ significantly decreased according to an increase in PMA ( $\rho=-0.491, p<0.001$ and $\rho=-0.257, p=0.017$, respectively) (Figure $1 \mathrm{~A}$ and $1 \mathrm{C}$ ). Consistently, nRR significantly increased by increasing PMA during both invasive and noninvasive NAVA ( $\rho=0.428, p<0.001$ and $\rho=0.225, p=0.038$, respectively) (Figure 1B and 1D). During invasive NAVA, the median time on backup ventilation decreased from $25.1 \% / \mathrm{min}$ for infants below $26^{+0}$ weeks PMA to less than $15 \% / \mathrm{min}$ for infants above $26^{+0}$ weeks PMA. The median nRR increased from 33.0 breaths $/ \mathrm{min}$ for infants below $26^{+0}$ weeks PMA to more than 45 breaths/min in infants above $26^{+0}$ weeks PMA. Several outliers were found in both percentage time on backup ventilation and nRR in most PMA groups (Figure $1 \mathrm{~A}$ and $1 \mathrm{~B})$. During noninvasive NAVA, the median time on backup ventilation decreased from $5.6 \% / \mathrm{min}$ to less than $3 \% / \mathrm{min}$, and the median nRR increased from 45 breaths/min to more than 55 breaths/min for infants below $26^{+0}$ weeks and above $30^{+0}$ weeks PMA (Figure $1 \mathrm{C}$ and $1 \mathrm{D}$ ).

There were 7 episodes of clinical deterioration in 6 patients. The episodes were caused by hemodynamically significant patent ductus arteriosus, clinical sepsis, ventilator-associated pneumonia and necrotizing enterocolitis (Table 2). Relative backup ventilation significantly increased up to the episode of clinical deterioration (Figure 2). The percentage of backup ventilation increased by $1.3(0.8-2.0)$ fold from 24 to 18 hours, 1.7 (1.2-3.1) fold from 18 to 12 hours, 2.0 (1.3-4.6) fold from 12 to 6 hours, and 3.4 (2.3-6.4) fold from 6 to 0 hours prior to the episodes compared to the baseline (Friedman test's $\chi^{2}=20.69, p<0.001$ ). After stabilization, all 6 infants returned to NAVA support.

Except for the 7 episodes described above, ventilatory support with invasive NAVA continued without interruptions until extubation. Noninvasive NAVA was changed to invasive NAVA if the infant needed intubation or discontinued when the infant stabilized to cope with nasal continuous positive airway pressure or high-flow nasal cannula.

\section{Discussion}

This study demonstrated that the proportion of backup ventilation decreased with increasing gestational age, especially after 26 weeks PMA. However, even the most immature infants triggered most of the ventilator breaths by their own respiration. An increase in the proportion of backup breaths anticipated clinical deteriorations, thereby showing the clinical value of observing the trends of backup ventilation.

Preterm infants have immature respiratory control leading to apnea. ${ }^{15,16}$ With increasing myelination of the brainstem, respiratory effort becomes stronger, and the number of apnea decreases. ${ }^{15-17}$ Postnatal maturation of the respiratory center explains our results: the decrease in backup ventilation and the increase in $\mathrm{nRR}$ with increasing gestational age. Our study showed that nRRs were already in the physiologic range of $40-$ 60 breaths per minute, and the proportion of backup breaths was very low from 26 weeks of gestation. This justifies the implementation of NAVA from a very early PMA.

The development of neural respiratory control could be demonstrated because the study population was supported with NAVA rather than other controlled ventilatory modes. In previous studies, controlled mechanical ventilation led to diaphragmatic dysfunction and prolonged ventilation, whereas patient-triggered ventilation prevented diaphragm weakness and maintained spontaneous breathing activity. ${ }^{18-22}$ Since NAVA supports infant respiration synchronized with the Edi and provides backup ventilation only during neural apnea, it would be ideal for preventing diaphragm weakness and strengthening their respiratory effort.

This is the first study to provide information about the typical amount of backup ventilation in preterm infants at different gestational ages. However, the amount of backup ventilation is affected by a set apnea time and a backup rate during NAVA. Choosing an optimal apnea time and a backup rate is important to avoid hypo-/hypercarbia in premature infants. ${ }^{23}$ These parameters should be chosen and adjusted according to each infant's age and clinical condition. In our study, the median apnea time was 3 and 5 seconds for invasive and noninvasive NAVA, respectively, and the median backup rate was 30 breaths per minute for 
both. The included infants remained stable with these settings except during their clinical deteriorations.

As the NAVA mode is based on the patient's own respiratory drive, it is inevitably sensitive to detect an increase in apnea, which is the most common symptom of clinical deterioration in preterm infants. The current ventilators operating NAVA display trends in the proportion of backup ventilation and nRR for the last 24 or 72 hours. These trends can be useful to detect early signs of clinical deterioration in this vulnerable population.

This study has weaknesses inherent to observational studies. First, \souteach PMA group was heterogeneous in terms of the severity of respiratory distress and length of mechanical ventilation. However, our sample came from approximately 1 year of patient days (354) and included almost half of all ventilated preterm infants in a level III neonatal intensive care unit during the study period. Second, because we collected data for mechanically ventilated patients, the majority of study recordings was from infants having bronchopulmonary dysplasia, and they do not represent all preterm neonates. However, since they are the target population of ventilator care, it is meaningful to understand the developmental changes in their neural respiratory drive and the significance of an acute increase in backup ventilation.

In conclusion, we used the proportion of backup ventilation during NAVA to show how the control of breathing matured with increasing gestational age in preterm infants. It is important that even the most immature infants triggered most of their breaths by their own respiration. As an acute increase in the proportion of backup ventilation anticipated clinical deterioration, we identified the trends in backup ventilation as a useful tool to detect early warning signs of clinical deterioration in preterm infants.

\section{Acknowledgements}

The authors would like to thank the Baby Friendly Ventilation Study Group. We are also grateful to all the parents, nurses and medical staff of the neonatal intensive care unit of Turku University Hospital for their willingness to assist with the study.

Author contributions: JL conceptualized and designed the study, collected data, carried out analyses and drafted the manuscript. VP collected data, reviewed and revised the manuscript. LL reviewed and revised the manuscript for important intellectual content. HS participated in the design of the study, collected data, reviewed and revised the manuscript for important intellectual content. All authors read and approved the final version of the manuscript.

Conflict of interest: The authors declare no competing interests.

Patient consent: Informed parental consents were obtained prior to study participation and the study was approved by the Ethics Committee of Hospital District of Southwest Finland (Approval number TO8/004/20).

ClinicalTrials.gov registration number : NCT04659083

Data availability statement: The data that support the findings of this study are available from the corresponding author upon reasonable request.

\section{References}

1. Sinderby C, Navalesi P, Beck J, Skrobik Y, Comtois N, Friberg S, Gottfried SB, Lindström L. Neural control of mechanical ventilation in respiratory failure. Nat Med . 1999;5:1433-1436.

2. Brander L, Howard LP, Beck J, Brunet F, Hutchison SJ, Slutsky AS, Sinderby C. Titration and implementation of neurally adjusted ventilatory assist in critically III patients. Chest . 2009;135:695-703.

3. Sinderby C, Beck J, Spahija J, De Marchie M, Lacroix J, Navalesi P, Slutsky AS. Inspiratory muscle unloading by neurally adjucted ventilatory assist during maximal inspiratory efforts in healthy subjects. Chest . 2007;131:711-717. 
4. Lee J, Kim HS, Sohn JA, Lee JA, Choi CW, Kim EK, Kim BI, Choi JH. Randomized crossover study of neurally adjusted ventilatory assist in preterm infants. J Pediatr . 2012;161:808-813.

5. Bates ML, Pillers D-AM, Palta M, Farrell ET, Eldridge MW. Ventilatory control in infants, children, and adults with bronchopulmonary dysplasia. Respir Physiol Neurobiol . 2013;189:329-337.

6. Lee J, Kim HS, Jung YH, Shin SH, Choi CW, Kim EK, Kim BI, Choi JH. Non-invasive neurally adjusted ventilatory assist in preterm infants: a randomised phase II crossover trial. Arch Dis Child Fetal Neonatal Ed . 2015;100:F507-F513.

7. Longhini F, Ferrero F, De Luca D, Cosi G, Alemani M, Colombo D, Cammarota G, Berni P, Conti G, Bona $\mathrm{G}$, et al. Neurally adjusted ventilatory assist in preterm neonates with acute respiratory failure.Neonatology . 2015;107:60-67.

8. Stein H, Howard D. Neurally adjusted ventilatory assist in neonates weighing < 1500 grams: A retrospective analysis. J Pediatr . 2012;160:786-789.

9. Kraaijenga JV, de Waal CG, Hutten GJ, de Jongh FH, van Kaam AH. Diaphragmatic activity during weaning from respiratory support in preterm infants. Arch Dis Child Fetal Neonatal Ed . 2017;102:F307F311.

10. Beck J, Reilly M, Grasselli G, Mirabella L, Slutsky AS, Dunn MS, Sinderby C. Patient-ventilator interaction during neurally adjusted ventilatory assist in low birth weight infants. Pediatr Res . 2009;65:663-668.

11. Harris J, Tibby SM, Endacott R, Latour JM. Neurally adjusted ventilatory asist in infants with acute respiratory failure: a literature scoping review. Pediatr Crit Care Med . 2021;online ahead of print

12. Biban P, Serra A, Polese G, Soffiati M, Santuz P. Neurally adjusted ventilatory assist: a new approach to mechanically ventilated infants.J Matern Fetal Neonatal Med . 2010;23 Suppl 3:38-40.

13. Vignaux L, Grazioli S, Piquilloud L, Bochaton N, Karam O, Jaecklin T, Levy-Jamet Y, Tourneux P, Jolliet P, Rimensberger PC. Optimizing patient-ventilator synchrony during invasive ventilator assist in children and infants remains a difficult task*. Pediatr Crit Care Med . 2013;14:e316-e325.

14. Baudin F, Wu H-T, Bordessoule A, Beck J, Jouvet P, Frasch MG, Emeriaud G. Impact of ventilatory modes on the breathing variability in mechanically ventilated infants. Front Pediatr . 2014;2:132.

15. Abu-Shaweesh JM, Martin RJ. Neonatal apnea: what's new? Pediatr Pulmonol . 2008;43:937-944.

16. Mathew OP. Apnea of prematurity: pathogenesis and management strategies. J Perinatol . 2011;31:302310.

17. Martin RJ, Abu-Shaweesh JM. Control of breathing and neonatal apnea.Biol Neonate . 2005;87:288-295.

18. Jung B, Constantin JM, Rossel N, Le Goff C, Sebbane M, Coisel Y, Chanques G, Futier E, Hugon G, Capdevila $\mathrm{X}$, et al. Adaptive support ventilation prevents ventilator-induced diaphragmatic dysfunction in piglet: an in vivo and in vitro study. Anesthesiology . 2010;112:1435-1443.

19. Goligher EC, Laghi F, Detsky ME, Farias P, Murray A, Brace D, Brochard LJ, Sebastien-Bolz S, Rubenfeld GD, Kavanagh BP, et al. Measuring diaphragm thickness with ultrasound in mechanically ventilated patients: feasibility, reproducibility and validity. Intensive Care Med . 2015;41:642-649.

20. Goligher EC, Fan E, Herridge MS, Murray A, Vorona S, Brace D, Rittayamai N, Lanys A, Tomlinson G, Singh JM, et al. Evolution of diaphragm thickness during mechanical ventilation: impact of inspiratory effort. Am J Respir Crit Care Med . 2015;192:1080-1088.

21. Goligher EC, Dres M, Fan E, Rubenfeld GD, Scales DC, Herridge MS, Vorona S, Sklar MC, Rittayamai $\mathrm{N}$, Lanys A, et al. Mechanical ventilation-induced diaphragm atrophy strongly impacts clinical outcomes. Am J Respir Crit Care Med . 2018;197:204-213. 
22. Jaber S, Petrof BJ, Jung B, Chanques G, Berthet JP, Rabuel C, Bouyabrine H, Courouble P, KoechlinRamonatxo C, Sebbane M, et al. Rapidly progressive diaphragmatic weakness and injury during mechanical ventilation in humans. Am J Respir Crit Care Med . 2011;183:364-371.

23. Morgan EL, Firestone KS, Schachinger SW, Stein HM. Effects of changes in apnea time on the clinical status of neonates on NIV-NAVA.Respir Care . 2019;64:1096-1100.

\section{Figure legends}

Figure 1. Time percentage on backup ventilation and neural respiratory rate (nRR) according to postmenstrual age during invasive and noninvasive neurally adjusted ventilatory assist (NAVA).

Spearman test was used for the analyses. For invasive NAVA, each PMA group included 17, 22, 52, 60, 66, 43 and 9 infants. For noninvasive NAVA, each PMA group included 9, 8, 9, 35 and 24 infants. Box plots show median (IQR), minimum and maximum values.

Figure 2. Relative ratio of time on backup ventilation compared to the baseline of 24 hours before the episode (-24hours).

Values are medians (IQRs) of the previous 6 hours. Grey lines indicate individual changes. Values at each time point are $1.3(0.8-2.0), 1.7(1.2-3.1), 2.0(1.3-4.6)$ and $3.4(2.3-6.4)$, respectively. Friedman test's $\chi^{2}$ was $20.69(p<0.001)$.

\section{Hosted file}

BU_Table_20210514.pdf available at https://authorea.com/users/414420/articles/522456-backupventilation-during-neurally-adjusted-ventilatory-assist-in-preterm-infants
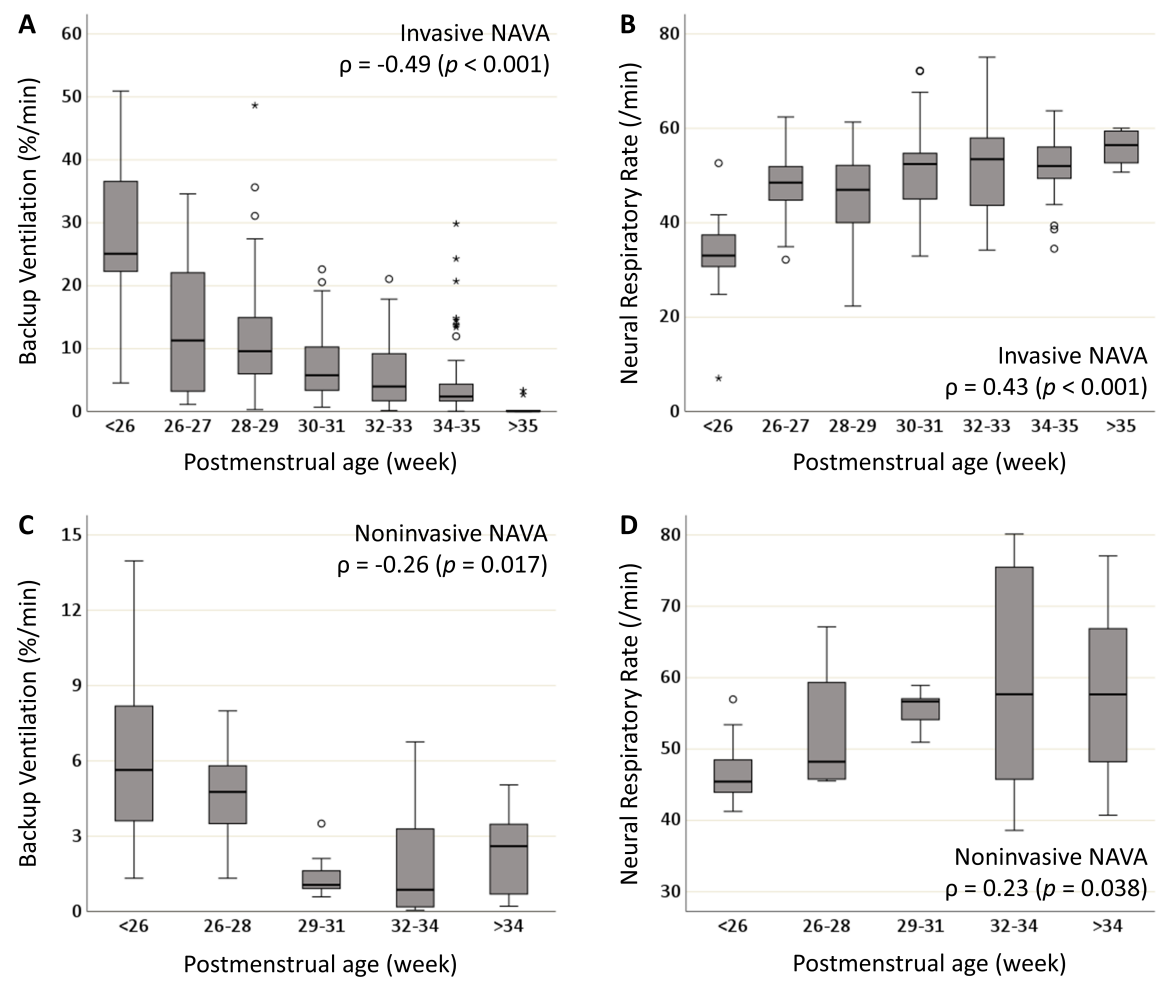

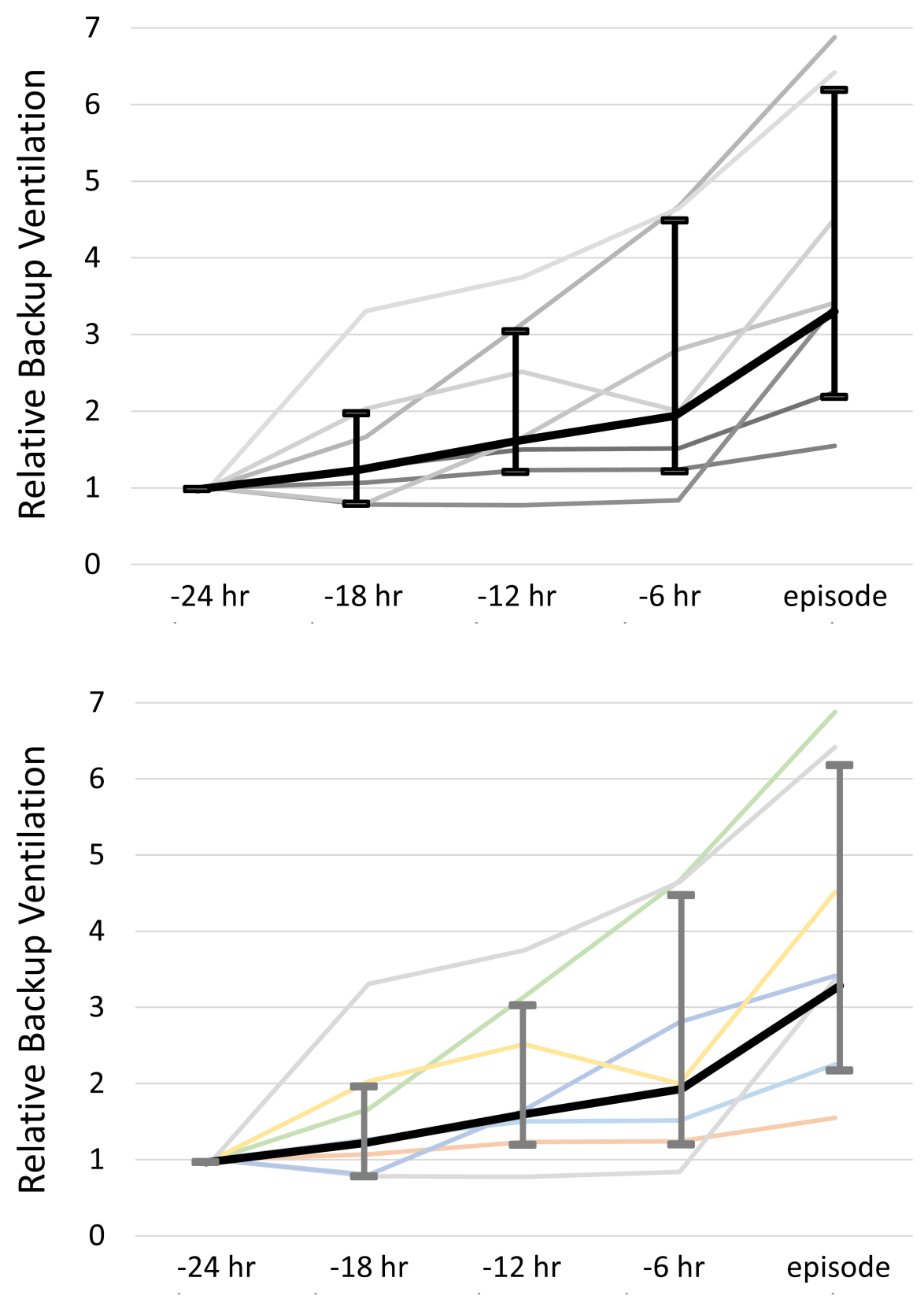